\title{
Excess of topological defects induced by confinement in vortex nanocrystals
}

\author{
N. R. Cejas Bolecek, ${ }^{1}$ M. I. Dolz, ${ }^{2}$ H. Pastoriza, ${ }^{1}$ M. Konczykowski, ${ }^{3}$ C. J. van der Beek, ${ }^{3}$ A. B. Kolton, ${ }^{4}$ and Y. Fasano ${ }^{1, *}$ \\ ${ }^{1}$ Laboratorio de Bajas Temperaturas, Centro Atómico Bariloche \& Instituto Balseiro, Bariloche, Argentina \\ ${ }^{2}$ Departamento de Física, Universidad Nacional de San Luis, CONICET, Argentina \\ ${ }^{3}$ Laboratoire des Solides Irradiés, École Polytechnique, CNRS, CEA, Université Paris-Saclay, Palaiseau, France \\ ${ }^{4}$ Teoría de la Materia Condensada, Centro Atómico Bariloche \& Instituto Balseiro, Bariloche, Argentina
}

(Received 27 March 2017; revised manuscript received 16 June 2017; published 14 July 2017)

\begin{abstract}
We directly image individual vortex positions in nanocrystals in order to unveil the structural property that contributes to the depletion of the entropy jump entailed at the first-order transition. On reducing the nanocrystal size, the density of topological defects increases near the edges over a characteristic length. Within this "healinglength" distance from the sample edge, vortex rows tend to bend, while towards the center of the sample, the positional order of the vortex structure is what is expected for the Bragg-glass phase. This suggests that the healing length may be a key quantity to model confinement effects in the first-order transition of extremely layered vortex nanocrystals.
\end{abstract}

DOI: 10.1103/PhysRevB.96.024507

\section{INTRODUCTION}

The growing demand of miniaturization in superconducting devices applied to the detection of different types of radiation [1-5] and magnetic signals [6-8] has triggered the study of thermodynamic and transport properties of high- $T_{\mathrm{c}}$ superconducting materials at the micro- and nanoscale. Since many of these devices operate in the mixed state, understanding the change in thermodynamic and structural properties of vortex matter when reducing the number of vortices down to the nanoscale is crucial for predicting their working range. For instance, transition-edge superconducting devices are based on the detection of a sudden increase of dissipation due to particular events when continuously measuring voltage with a low applied current. If confinement changes the critical current up to which vortices do not dissipate, then the detection edge needs to be readjusted.

From a fundamental point of view, vortex matter in type-II superconductivity is a case study for understanding how the physical properties and phase diagrams change when going from macroscopic to nanocrystalline condensed matter. In the case of hard condensed matter, nanocrystals are made up of, at most, a few-thousand particles (atoms) [9,10]; similarly, soft-condensed-matter "vortex nanocrystals" can be nucleated in micron-sized samples with the same amount of vortices [11-18]. Typically, hard-condensed-matter nanocrystals present a decrease of transition temperatures, entropy, and enthalpy jumps in melting and solid-solid first-order phase transitions $[9,10,19,20]$. This is the consequence of a depletion of the total binding energy since the particle's surface-tovolume ratio increases on decreasing the system size. In the case of nanocrystalline vortex matter, confinement effects also affect the transition lines and structural properties [21,22], although different degrees of freedom and interactions are at play. Particularly, in the case of vortex nanocrystals nucleated in extremely layered high-temperature superconductors, the phase diagram is finely tuned by many energy scales [23]. The interplay among intervortex interactions, thermal fluctuations,

\footnotetext{
*Corresponding author: yanina.fasano@cab.cnea.gov.ar
}

pinning, and extremely anisotropic magnetic properties can be controlled by applied field, temperature, crystalline disorder, and oxygen doping, respectively.

Recently, some of us reported [24] on the peculiarities of decreasing the system size in extremely layered vortex matter: for roughly one-hundred particles (vortices), no melting-point depression is observed, in contrast to results in hard condensed matter [9]. The entropy jump entailed at the first-order transition decreases on reducing the system size and we suggested that this might have its origin in two effects that can eventually occur simultaneously at the transition. First, since there is evidence that the first-order transition might be concomitant with a $c$-axis decoupling of pancake vortices [25], confinement can induce an extra contribution to decoupling and thus reduce the entropy jump. Second, confinement can also entail a deterioration of the in-plane structural order when nucleating vortex nanocrystals [24]. Unveiling the structural properties of vortex nanocrystals is therefore mandatory in order to gain insight and model the origin of the entropy-jump depletion.

In this work, we study the evolution of the structural properties on reducing the system size of nanocrystalline vortex matter. We characterize the variation and spatial distribution of elastic and plastic deformations in the quenched nanocrystalline vortex solid. Our vortex nanocrystals with less than 4000 vortices are nucleated in the whole area of micron-sized engineered samples at low applied fields. We have direct access to the static structural properties with single-vortex resolution [26]. We present a systematic study as a function of the number of vortices in the nanocrystal, tuned either by sample physical size or vortex density.

\section{EXPERIMENT}

Nanocrystalline vortex matter is nucleated in micronsized $\mathrm{Bi}_{2} \mathrm{Sr}_{2} \mathrm{CaCu}_{2} \mathrm{O}_{8+y}$ disks with diameters $d$ in the range $30-50 \mu \mathrm{m}$ and thicknesses between 1 and $2 \mu \mathrm{m}$. Disks are engineered from optimally doped crystals $\left(T_{\mathrm{c}}=90 \mathrm{~K}\right)$ by combining optical-lithography and physical ion-milling techniques [27]. During the last step of the sample fabrication process, thin freestanding and freshly cleaved disks are 
obtained. The micron-sized samples were obtained from the macroscopic sample studied in Ref. [28], and part of the fabricated disks were used to investigate the phase diagram of mesoscopic vortex matter reported in Ref. [24]. The disks are placed on the sample holder with micromanipulators and carefully glued with conducting epoxy such that the cleaved surfaces remain clean.

As a result of the final cleaving process, more than $90 \%$ of the disks present submicron steps at the surface. We avoid using these disks for our study when possible. In some cases, we considered disks with submicron steps that divide the sample in terraces with one of them containing less than $\sim 10 \%$ of vortices. In these cases, we only consider for our analysis the roughly $90 \%$ of vortices that are at the same terrace.

The nanocrystalline vortex solid is directly imaged with single-vortex resolution by magnetically decorating vortex positions at $4.2 \mathrm{~K}$ after field cooling the sample at low fields from $T>T_{\mathrm{c}}$, as described in Ref. [29]. The lattice spacing of the vortex structure, $a=1.075 \sqrt{\Phi_{0} / B}$, was tuned by changing the applied field that controls the magnetic induction $B$. For the experiments presented here, $a$ ranges from 0.7 to $1.2 \mu \mathrm{m}$ ( $B$ from 50 to $16 \mathrm{G}$ ). These lattice-spacing values are well above the zero-temperature penetration depth for this material, $\lambda(0)=0.2 \mu \mathrm{m}$ [23]. In field-cooling experiments, the vortex structure decorated at $4.2 \mathrm{~K}$ corresponds to a configuration frozen at length scales of lattice spacing at a larger temperature, $T_{\text {freez }}$ [29]. The magnetic decoration technique is not sensitive to the vortex meandering at distances smaller than $\lambda$ that can occur at $T<T_{\text {freez }}$. For the material studied here, $T_{\text {freez }} \sim T_{\text {irr }}$, the irreversibility temperature at which pinning sets in [30] $\left(T_{\text {irr }} \sim 85 \mathrm{~K}\right.$ for the studied field range [28]).

\section{RESULTS}

Figure 1 shows real-space images of vortex nanocrystals with a vortex density of $16 \mathrm{G}$ nucleated in micron-sized $\mathrm{Bi}_{2} \mathrm{Sr}_{2} \mathrm{CaCu}_{2} \mathrm{O}_{8+\delta}$ disks with $d$ ranging 50 to $30 \mu \mathrm{m}$ (with approximately 1500 to 400 vortices). The nanocrystals have the outer vortex compact lines slightly bent, following the edges of the samples. In all cases, this effect is produced without a detectable change in vortex density (within 2\%) in the whole vortex nanocrystal. Vortices at the center of the sample form a crystallite with decreasing size on reducing field. As observed in Fig. 1, these two structural properties are achieved by accumulating plastic deformations towards the edges of the nanocrystals. This is evident in the Delaunay triangulations [30] of the right panels that indicate the nonsixfold coordinated vortices, highlighted in red, and the neighborhood of plastic deformations, highlighted in gray. These topological defects are mainly unpaired screw dislocations, each formed by a five- and a seven-fold coordinated vortex. The Fourier transforms of the central panels of Fig. 1 show six diffraction peaks that broaden on decreasing sample size. For the smallest studied sample, these peaks split up due to the nucleation of two crystallites of similar size with compact planes with a very small misalignment (smaller than $5^{\circ}$ ); see Fig. 1(c). Therefore, plastic deformations producing topological defects in the vortex nanocrystal proliferate on increasing confinement effects for a fixed vortex density.

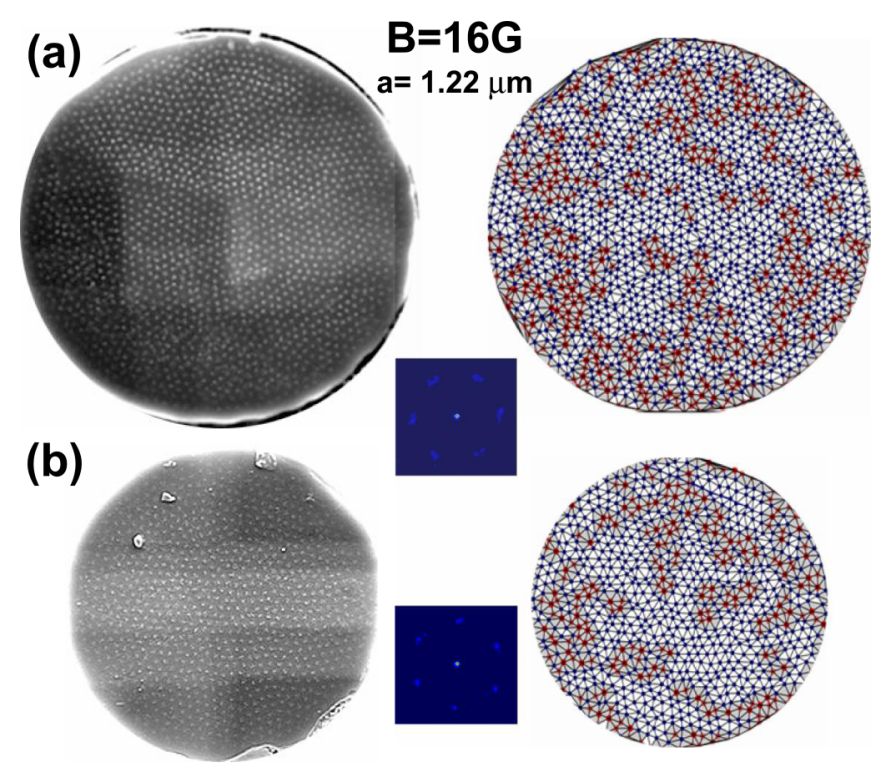

(c)

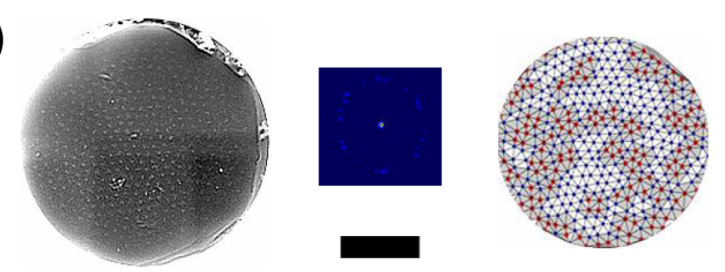

FIG. 1. Vortex nanocrystals with a vortex density of $16 \mathrm{G}$ nucleated in micron-sized $\mathrm{Bi}_{2} \mathrm{Sr}_{2} \mathrm{CaCu}_{2} \mathrm{O}_{8+\delta}$ disks with diameters of (a) 50, (b) 40, and (c) $30 \mu \mathrm{m}$. Left panels: Vortices imaged in white by means of field-cooling magnetic decorations performed at $4.2 \mathrm{~K}$. Central panels: Fourier transforms of the vortex positions. Right panels: Delaunay triangulations of the vortex structure depicting nonsixfold (sixfold) coordinated vortices in red (blue) with plastic deformations highlighted in gray. The scale bar indicates $10 \mu \mathrm{m}$.

When increasing the vortex density to roughly two and three times $(B=32$ and $50 \mathrm{G})$, the outer vortex shells of the nanocrystals also mimic the edges of the sample without any noticeable local change in $a$; see Supplemental Material [31]. For the higher vortex densities of 32 and $50 \mathrm{G}$, the nanocrystal is formed by a single crystal in the whole sample in contrast to the central crystallites observed at $16 \mathrm{G}$. A particular case is that of the $32 \mathrm{G}$ vortex structure nucleated in a $50-\mu \mathrm{m}$-diameter disk presenting a submicron step at the sample surface. This feature induces a local ordering of the vortex structure presenting one compact plane parallel to the step in a region of less than $10 a$. As a result, a planar grain boundary of paired screw dislocations is formed in the nanocrystal. Besides this spurious effect, on enhancing the nanocrystal stiffness (by increasing $B$ [23]), the stress induced by the outer vortex shells mimicking the sample edge produces a proliferation of isolated clusters of topological defects. The size of the clusters apparently increases with stiffness; see Supplemental Material [31].

In order to quantify the impact of pinning on increasing confinement, we will consider the distance evolution of the average displacement correlator. This magnitude defined as $W(r)=$ $\left\langle[u(r)-u(0)]^{2}\right\rangle / 2$ quantifies the average over quenched disorder and thermal fluctuations of the displacements of vortices 
with respect to the sites of a perfect triangular lattice, $u(r)$. In the case of macroscopic vortex matter, the theoretical prediction [32] states that the displacement correlator presents three different regimes as a function of $r / a$. Within the Larkin regime expected at short distances, $W(r) \propto r$ and the pinning is not yet effective in generating lattice distortions. When the displacements reach the scale in which pinning is effective, i.e., when $[u(r)-u(0)] \sim \xi$, the system is in the random manifold regime. In this regime, the evolution of the displacement correlator is algebraic with distance, $W(r) \propto r^{v}$ with $v=0.44$ for three-dimensional structures. In addition, in this regime, a constant ratio of transversal-to-longitudinal displacement correlators is expected, $W^{\mathrm{T}}(r) / W^{\mathrm{L}}(r) \sim 1.44$. The longitudinal correlation function is defined as $W_{\mathrm{L}}(r)=$ $\left\langle[\mathbf{u}(r)-\mathbf{u}(0)] \cdot \mathbf{r} / r^{2}\right\rangle / 2$. The displacements perpendicular to the vortex structure's main directions are quantified by the transverse displacement correlator that can be obtained as $W_{\mathrm{T}}=2 W(r)-W_{\mathrm{L}}(r)$. For sufficiently large distances such that $[u(r)-u(0)]>a$, the Bragg-glass structure enters in the quasiordered regime in which the dependence of $W(r)$ is logarithmic with distance.

Direct imaging of vortex structures in dislocation-free regions of $\mathrm{Bi}_{2} \mathrm{Sr}_{2} \mathrm{CaCu}_{2} \mathrm{O}_{8+\delta}$ vortex matter [33] showed the stabilization of the Larkin and random manifold regimes. The Bragg-glass regime was not directly observed since at distances larger than $100 a$, the structure presents topological defects and thus $u(r)$ is not well defined. In order to estimate $W(r)$ in our vortex nanocrystals, we implemented an algorithm for locally calculating the displacement correlator in the presence of topological defects. The algorithm calculates $W(r)$ not in the whole vortex nanocrystal but in regions, considering lanes of vortices in the three principal directions of the structure. The regional lanes stop running two lattice parameters away of any topological defect. The schematic representation of Fig. 2 indicates how the lanes are defined in a given vortex structure and how $W(r)$ is calculated for every lane. The figure also illustrates the lanes (color lines) identified, for instance, in a $16 \mathrm{G}$ vortex nanocrystal nucleated in a 50- $\mu \mathrm{m}$-diameter disk. The algorithm computes the $W_{k}^{i}(r)$,with $k=1,2,3$ the three principal directions of the structures, up to a distance equal to the length of every $i$ th lane. Then we average the results obtained for all the $i$ th parallel lanes within the $k$ direction in order to obtain $W_{k}$. Finally, we calculate $W^{*}$ by averaging the three $W_{k}$ magnitudes associated to the main directions. We present the $W^{*}$ data for macroscopic and nanocrystalline vortex matter for different sample sizes and applied fields of 16,32 , and $50 \mathrm{G}$ in Fig. 3.

For macroscopic $\mathrm{Bi}_{2} \mathrm{Sr}_{2} \mathrm{CaCu}_{2} \mathrm{O}_{8+\delta}$ vortex matter with a density of $16 \mathrm{G}$, we do not observe the Larkin regime $W^{*}(r) \sim r$, indicating that the Larkin length is smaller than our spatial resolution for $u(r)$. We do observe, however, that $W^{*}(r) \sim(r / a)^{v}$ with $v=(0.4 \pm 0.1)$ and $W^{\mathrm{T}}(r) / W^{\mathrm{L}}(r) \sim$ $(1.4 \pm 0.5)$ up to the $r / a \sim 10$ range [see Fig. 3(a)], as theoretically expected for an equilibrated random-manifold regime. In the case of the more dense macroscopic vortex structures of 32 and $50 \mathrm{G}$, we find the same exponent for the algebraic dependence of $W^{*}(r)$ within 5\% dispersion for such a short-distance scale. Therefore, vortex structures in macroscopic samples are in agreement with the nucleation of
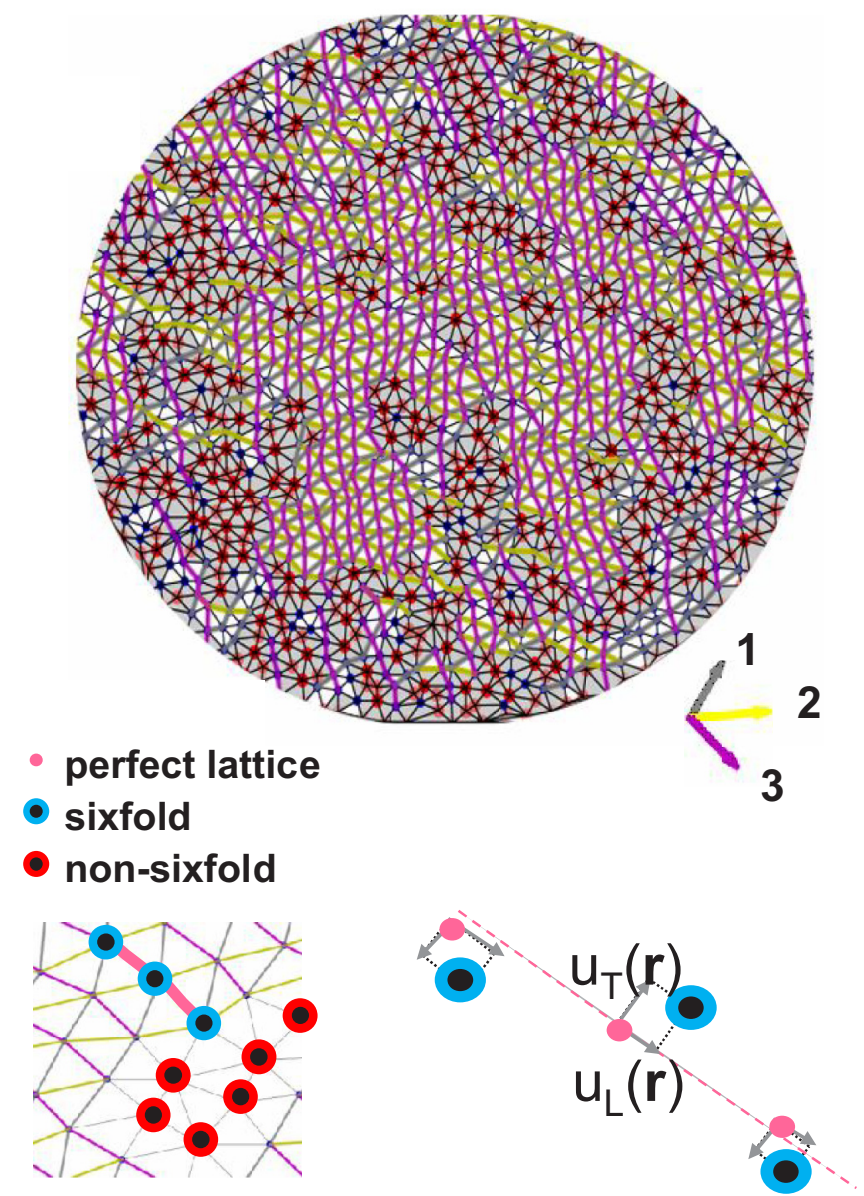

FIG. 2. Top: Lanes considered to calculate the local vortex displacements $u(r)$ in the case of the $16 \mathrm{G}$ vortex nanocrystal nucleated in the $50 \mu \mathrm{m}$ disk of Fig. 1(a). The lanes running parallel to one of the three principal directions of the vortex structure are identified with the same color. Bottom: Schematics of the vortex displacements computed in order to calculate the displacement correlator $W^{*}(r)$ along $\left[u_{\mathrm{L}}(r)\right]$, and perpendicular to $\left[u_{\mathrm{T}}(r)\right]$, a given lane at a distance $r$ from its starting point. The real positions of the vortices are indicated with large dots, whereas the positions corresponding to a perfect triangular lattice are shown in small gray dots.

a Bragg-glass phase. Since the decorated structure is expected to be a snapshot of a configuration freezed at a characteristic temperature $T_{\text {freez }} \sim T_{\text {irr }}$ [22,30], our results yield information on creep-relaxation (random-manifold) dynamics at such a temperature. In particular, they show that at $T_{\text {freez }}$, length scales as large as $\sim 10 a$ and $5-15 \mu \mathrm{m}$ can get equilibrated in the experimental time scale.

This scale of equilibration of the random-manifold regime is of the order of the vortex nanocrystals that we study. The displacement correlator in nanocrystalline vortex matter, as a function of field and sample physical size, is shown in Figs. 3(b) and 3(c). The absolute value of $W^{*}(r) / a^{2}$ for vortex nanocrystals is more than $30 \%$ larger than for macroscopic samples. The $r / a$ evolution of the displacement correlator is algebraic even for the smallest nanocrystal with roughly 400 vortices. 


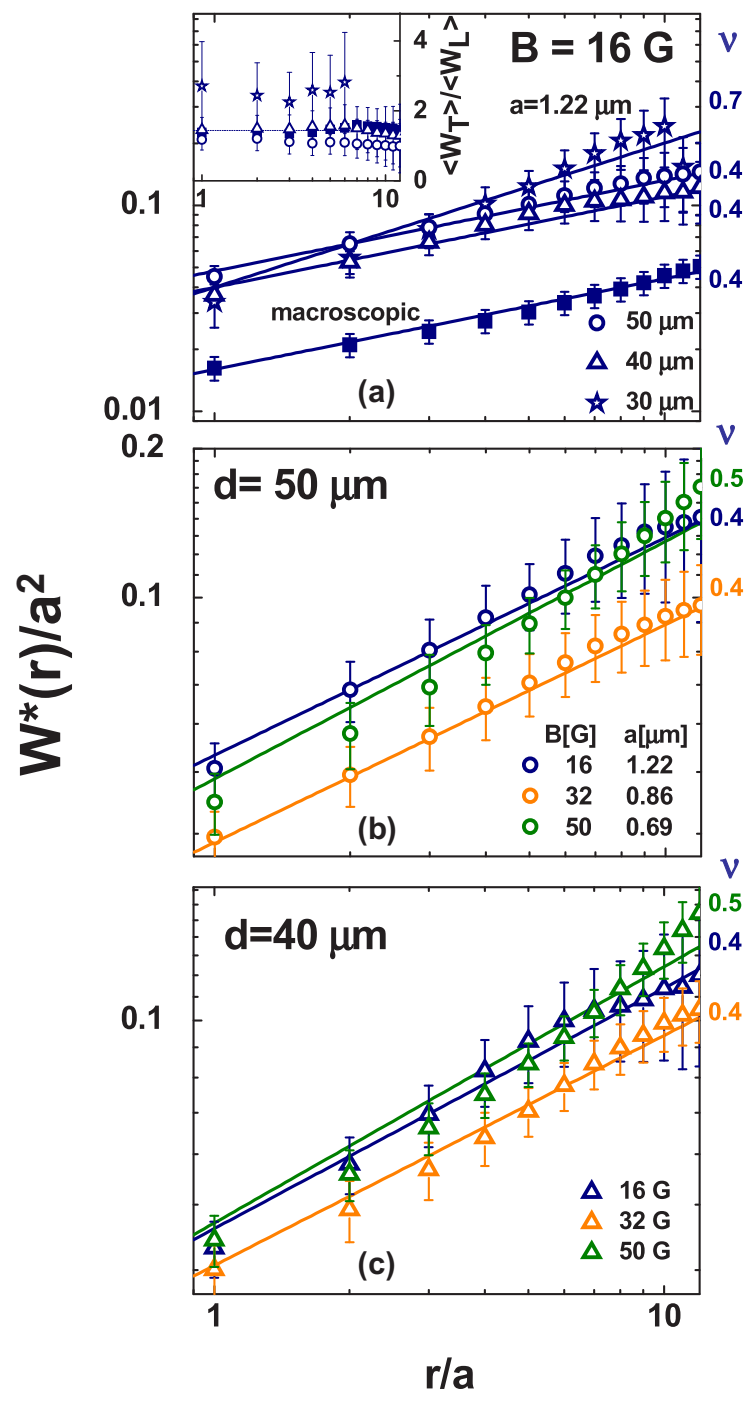

FIG. 3. Evolution of the normalized displacement correlator $W^{*}(r) / a^{2}$ with $r / a$ for vortex nanocrystals nucleated in micron-sized $\mathrm{Bi}_{2} \mathrm{Sr}_{2} \mathrm{CaCu}_{2} \mathrm{O}_{8+\delta}$ disks. (a) Data for $16 \mathrm{G}$ vortex structures nucleated in macroscopic samples (full squares) and disks (open symbols). The inset shows the transversal-to-longitudinal displacement correlator ratio and dashed lines indicate the 1.44 value expected theoretically. Magnetic field evolution of the displacement correlator for disks of (b) 50 and (c) $40 \mu \mathrm{m}$ diameters. Full lines are fits to the data with an algebraic decay with an exponent $v$ (with an error of \pm 0.1 ) indicated for each case at the right.

The exponent $v$ and the $W_{\mathrm{T}} / W_{\mathrm{L}}$ ratio have values expected for the random-manifold regime within the error, but in the case of the smallest $16 \mathrm{G}$ nanocrystal, both magnitudes increase considerably. Indeed, the Delaunay triangulation of Fig. 1(c) shows a structure with very small crystallites. When increasing the vortex density for the same sample size, the structure also presents an algebraic $W^{*}(r)$ with an exponent of roughly 0.4 within the error of 0.1 , whereas it slightly enhances on increasing vortex density; see Figs. 3(b) and 3(c). The increase of $v$ when enhancing confinement can be due to the bending of vortex rows close to the sample edge dominating the nucleation of the smallest studied nanocrystal.
In order to study these edge effects, we quantify the proliferation of topological defects with the radial density of topological defects, $\rho_{\text {def }}(r)=N_{\text {def }}(r) / N_{\mathrm{v}}(r)$. We actually consider $N_{\text {def }}$ and $N_{\mathrm{v}}$ at a radius $r$ as the number of defects and of vortices included in a circular shell with inner radius $r-\delta r / 2$ and outer radius $r+\delta r / 2$. The calculations are performed considering concentric circular shells of width $\delta r=$ $2 a$ and taking the origin at the center of the vortex nanocrystal. The top panel of Fig. 4 shows the spatial distribution of defects in the considered circular shells. Figure 4(a) shows $\rho_{\text {def }}(r)$ at a fixed vortex density of $16 \mathrm{G}$ for samples with three different radii, whereas Fig. 4(b) shows the results at densities of 16, 32 , and $50 \mathrm{G}$ for the same sample size of $50 \mu \mathrm{m}$. The error bars in the data correspond to the standard deviation of the values obtained in different magnetic decorations for the same sample diameter and $B$.

In all cases, $\rho_{\mathrm{def}}(r)$ increases dramatically on approaching the sample edge (indicated in the figures with dashed lines) and stagnates on the center of the nanocrystals. These saturation values roughly approach, within the dispersion of values for different experimental realizations, the density of defects found in macroscopic samples for each $B, \rho_{\text {def }}^{\text {macro }}=$ $N_{\text {def }}^{\text {macro }} / N_{\mathrm{v}}^{\text {macro }}$. For the macroscopic parent sample from which the disks were engineered, $\rho_{\text {def }}^{\text {macro }} \sim 11,3$, and $2 \%$ for 16,32 , and $50 \mathrm{G}$ structures, respectively [22] (see horizontal lines in Fig. 4). The total density of topological defects for the nanocrystals, $\rho_{\mathrm{def}}=N_{\mathrm{def}} / N_{\mathrm{v}}$, is always larger than $\rho_{\mathrm{def}}^{\text {macro }}$. For a fixed $B, \rho_{\text {def }}$ enhances on increasing confinement. On the other hand, for a fixed sample size, $\rho_{\mathrm{def}}$ increases when the structure softens on lowering $B$.

The abrupt increase of $\rho_{\mathrm{def}}(r)$ at the vicinity of the sample edge occurs in a larger typical distance when increasing the nanocrystal flexibility (decreasing $B$ ). We quantified this tendency by fitting the data with a $\rho_{\text {def }}(r)=A_{1}+$ $A_{2} \exp [(r / a-d / 2 a) / \alpha]$ dependence; see full lines in Fig. 4. The parameter $\alpha$ can be interpreted as a number of lattice parameters in which the vortex nanocrystal relaxes the shear stress induced by the bending of the outer vortex lines towards the center of the sample. This relaxation is performed via the nucleation of plastic deformations. We will thus regard $a \cdot \alpha$ as a characteristic "healing length." The evolution of $\alpha$ with $d$ for the three studied vortex densities is shown in Fig. 4(c). The length $\alpha$, in lattice parameter units, increases with sample size. This indicates that for larger nanocrystals, the nucleation of topological defects for mimicking the sample edge is performed more gradually than for smaller nanocrystals. In addition, for a fixed sample size, $\alpha$ increases on softening the vortex nanocrystal.

The previous analysis shows that the relevance of bulk pinning for vortex nanocrystals with 400-1500 vortices does not differ quantitatively from what is expected for macroscopic vortex matter. Physical properties that are affected by confinement in nanocrystalline vortex matter are thus controlled by the vortices located in a strip of width of the order of $a \cdot \alpha$ from the sample edges. The larger $a \cdot \alpha$ as compared to the sample diameter $d$, the more important are confinement effects. In summary, except for those cases in which more than $20 \%$ of vortices are located at the nanocrystal surface and therefore confinement effects are dominant, in the interior of samples, vortex nanocrystals nucleate and are able to locally equilibrate 

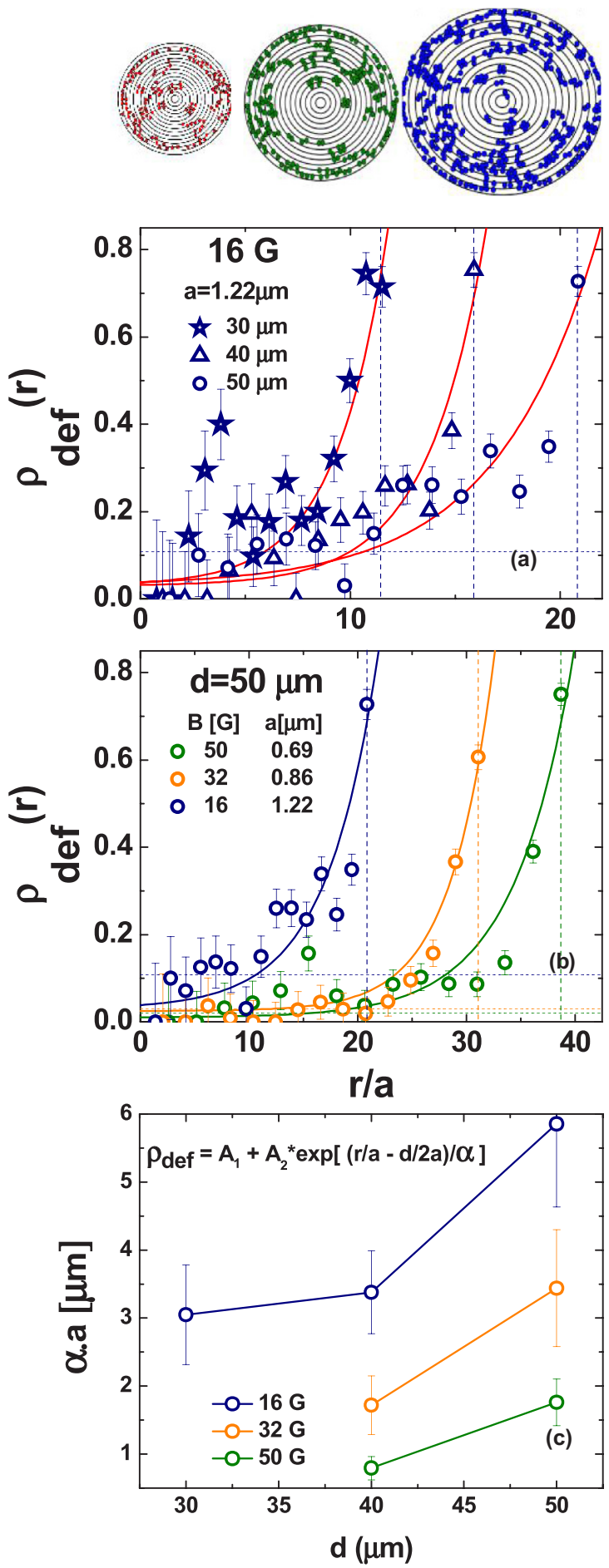

FIG. 4. Radial density of topological defects $\rho_{\text {def }}(r)$ as a function of $r / a$ for nanocrystalline $\mathrm{Bi}_{2} \mathrm{Sr}_{2} \mathrm{CaCu}_{2} \mathrm{O}_{8+\delta}$ vortex matter. (a) Vortex nanocrystals with a density of $16 \mathrm{G}$ nucleated in 30,40 , and $50 \mu \mathrm{m}$ diameter disks. Top panel: Circular shells considered to calculate this magnitude and location of topological defects (indicated with dots). (b) Vortex nanocrystals with densities of 16,32, and $50 \mathrm{G}$ nucleated in 50- $\mu \mathrm{m}$-diameter disks. Vertical dashed lines indicate the sample edge, $d / 2$, and horizontal lines indicate the topological defect density for the parent macroscopic sample, $\rho_{\mathrm{def}}^{\text {macro }}$. The full lines are fits to the data with a function $\rho_{\text {def }}(r)=A_{1}+A_{2} \exp [(r / a-d / 2 a) / \alpha]$. (c) Evolution of the healing length of vortex nanocrystals, $\alpha \cdot a$, with the sample diameter $d$ for the three studied vortex densities. a structure consistent with the Bragg-glass phase [32]. More precisely, for distances from the edge towards the center of the sample larger than $a \cdot \alpha$, nanocrystalline vortex matter is equilibrated in the random-manifold regime.

The healing length $a \cdot \alpha$ described above may be a useful quantity to model the $B$ and $d$-dependent entropy-jump depletion in the vortex first-order transition. This depletion refers to the observation of a smaller $\Delta S / \Delta S_{0}$ at the firstorder melting transition of nanocrystalline vortex matter as compared to the case of macroscopic samples; see inset to Fig. 4 in Ref. [24]. The entropy-jump depletion can have two extra contributions on top of the configurational one of the vortex solid phase. The first one can come from a change on the coupling of pancakes making up the outermost vortex lines when reducing the nanocrystal size. The second one can come from the possibility of the sample surface straightening the outermost vortices as any correlated disorder would produce. In any case, the existence of the characteristic length $a \cdot \alpha$ quantifying the crossover towards bulk behavior may be a useful empirical quantity for modeling these three plausible effects.

In this respect, it is worth noting that the value $\alpha$ we detect is finite and no appreciable spatial gradient in vortex density is observed in the decoration results presented here. This is in sharp contrast to the effects observed in other soft-condensed-matter systems such as disk-confined one-component plasmas, where the density is nonuniform and topological defects appear in the whole system [34]. Nevertheless, this finding qualitatively agrees with simulations for the case of systems with short-range interactions [35] which are more adequate to model vortex-vortex interactions in thick superconducting samples. However, unlike these theoretical case studies, vortices are line objects that interact with bulk point disorder and, in the case of vortex nanocrystals, they also interact with the sample surface that aligns the outermost vortices along its border and bends vortex rows. This makes our problem much more complex. In particular, (a) the expected equilibrium bulk order is not the crystalline Abrikosov order, and (b) the equilibration dynamics is expected to be glassy (much slower than for a clean system) such that the equilibrium may not be reached completely in a decoration experiment.

\section{CONCLUSION}

We have shown that confinement affects the structure of vortex nanocrystals nucleated in micron-sized $\mathrm{Bi}_{2} \mathrm{Sr}_{2} \mathrm{CaCu}_{2} \mathrm{O}_{8+\delta}$ samples producing an excess of topological defects within a characteristic healing-length distance from the edge. Towards the center of the nanocrystal, the vortex lattice recovers the bulklike structure observed in macroscopic samples, consistent with the nucleation of a Bragg-glass phase.

The existence of a finite healing length may help to explain why decreasing the system size to a few-hundred vortices does not induce any melting-point depression as observed for nanocrystals of hard condensed matter [10], but entails a progressive depletion of the entropy jump at the first-order transition [24]. Further experimental and theoretical investigations on the magnetic field, temperature, and samplesize dependence of the healing length would thus be promising in order to build a simple phenomenological model for the 
anomalous properties of small confined systems of vortices and alike.

\section{ACKNOWLEDGMENT}

We thank Ming Li for sample preparation.
[1] U. Welp, K. Kadowaki, and R. Kleiner, Nat. Photon. 7, 702 (2013).

[2] S. Sekimoto, C. Watanabe, H. Minami, T. Yamamoto, T. Kashiwagi, R. A. Klemm, and K. Kadowaki, Appl. Phys. Lett. 103, 182601 (2013).

[3] T. Kashiwagi, K. Nakade, B. Markovic, Y. Saiwai, H. Minami, T. Kitamura, C. Watanabe, K. Ishida, S. Sekimoto, K. Asanuma, T. Yasui, Y. Shibano, M. Tsujimoto, T. Yamamoto, J. Mirkovic, and K. Kadowaki, Appl. Phys. Lett. 104, 022601 (2014).

[4] T. Kashiwagi, K. Nakade, Y. Saiwai, H. Minami, T. Kitamura, C. Watanabe, K. Ishida, S. Sekimoto, K. Asanuma, T. Yasui, Y. Shibano, M. Tsujimoto, T. Yamamoto, B. Markovic, J. Mirkovic, R. A. Klemm, and K. Kadowaki, Appl. Phys. Lett. 104, 082603 (2014).

[5] C. Watanabe, H. Minami, T. Yamamoto, T. Kashiwagi, R. A. Klemm, and K. Kadowaki, J. Phys.: Condens. Matter 26, 172201 (2014).

[6] K. Takeda, H. Mori, A. Yamaguchi, H. Ishimoto, T. Nakamura, S. Kuriki, T. Hozumi, and S. Ohkoshi, Rev. Sci. Instrum. 79, 033909 (2008).

[7] A. Yamaguchi, K. Takeda, T. Matsumoto, and A. Sumiyama, J. Low Temp. Phys. 158, 704 (2010).

[8] T. Schwarz, J. Nagel, R. W'olbing, M. Kemmler, R. Kleiner, and D. Koelle, ACS Nano 7, 844 (2012).

[9] C. J. Coombes, J. Phys. F 2, 441 (1972).

[10] A. N. Goldstein, C. M. Echer, and A. P. Alivisatos, Science 256, 1425 (1992).

[11] A. K. Geim, I. V. Grigorieva, S. V. Dubonos, J. G. S. Lok, J. C. Maan, A. E. Filippov, and F. M. Peeters, Nature (London) 390, 259 (1997).

[12] R. Geurts, M. V. Milosevic, and F. M. Peeters, Phys. Rev. B 75, 184511 (2007).

[13] M. R. Connolly, M. V. Milosevic, S. J. Bending, and T. Tamegai, Phys. Rev. B 78, 132501 (2008).

[14] X. H. Chao, B. Y. Zhu, A. V. Silhanek, and V. V. Moshchalkov, Phys. Rev. B 80, 054506 (2009).

[15] T. Cren, L. Serrier-Garcia, F. Debontridder, and D. Roditchev, Phys. Rev. Lett. 107, 097202 (2011).

[16] P. J. Pereira, L. F. Chibotaru, and V. V. Moshchalkov, Phys. Rev. B 84, 144504 (2011).
[17] T. Tominaga, T. Sakamoto, T. Nishio, T. An, T. Eguchi, Y Yoshida, and Y. Hasegawa, J. Supercond. Nov. Magn. 25, 1375 (2012).

[18] S. A. Mills, C. Shen, Z. Xu, and Ying Liu, Phys. Rev. B 92, 144502 (2015).

[19] S. H. Tolbert and A. P. Alivisatos, Science 265, 373 (1994).

[20] G. Guisbiers and L. Buchaillot, Phys. Lett. A 374, 305 (2009).

[21] V. A. Schweigert and F. M. Peeters, Phys. Rev. B 57, 13817 (1998).

[22] N. R. Cejas Bolecek, M. I. Dolz, A. Kolton, H. Pastoriza, C. J. van der Beek, M. Konczykowski, M. Menghini, G. Nieva, and Y. Fasano, J. Low Temp. Phys. 179, 35 (2015).

[23] C. J. van der Beek, Thermodynamics of Vortices in Disordered Superconductors (European Universities Editions, 2010).

[24] M. I. Dolz, Y. Fasano, N. R. Cejas Bolecek, H. Pastoriza, V. Mosser, M. Li, and M. Konczykowski, Phys. Rev. Lett. 115, 137003 (2015).

[25] H. Pastoriza, M. F. Goffman, A. Arribere, and F. de la Cruz, Phys. Rev. Lett. 72, 2951 (1994).

[26] Y. Fasano, J. Herbsommer, and F. de la Cruz, Phys. Status Solidi B 215, 563 (1999).

[27] M. I. Dolz, A. B. Kolton, and H. Pastoriza, Phys. Rev. B 81, 092502 (2010).

[28] M. I. Dolz, Y. Fasano, H. Pastoriza, V. Mosser, M. Li, and M. Konczykowski, Phys. Rev. B 90, 144507 (2014).

[29] Y. Fasano, M. De Seta, M. Menghini, H. Pastoriza, and F. De la Cruz, Solid State Commun. 128, 51 (2003).

[30] Y. Fasano, M. De Seta, M. Menghini, H. Pastoriza, and F. de la Cruz, Proc. Nat. Acad. Sci. 102, 3898 (2005).

[31] See Supplemental Material at http://link.aps.org/supplemental/ 10.1103/PhysRevB.96.024507 for the structural properties of vortex nanocrystals at fields of 32 and $50 \mathrm{G}$.

[32] T. Giamarchi and P. Le Doussal, Phys. Rev. Lett. 72, 1530 (1994).

[33] P. Kim, Z. Yao, C. A. Bolle, and C. M. Lieber, Phys. Rev. B 60, R12589 (1999).

[34] A. Mughal and M. A. Moore, Phys. Rev. E 76, 011606 (2007).

[35] Ying-Ju Lai and Lin I, Phys. Rev. E 60, 4743 (1999). 